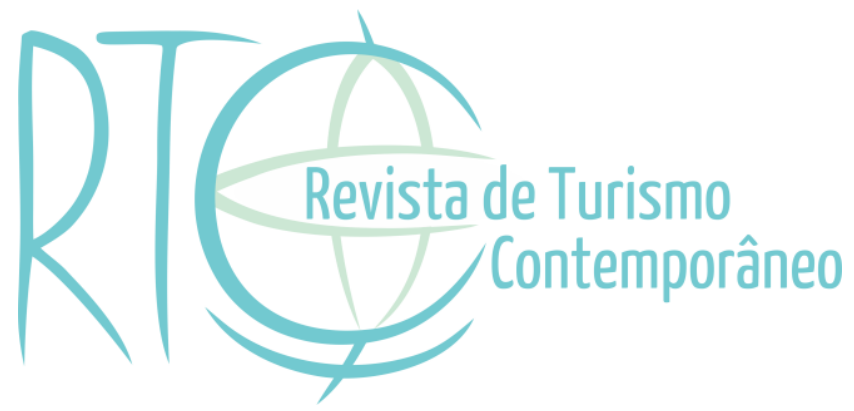

\title{
Metodologias utilizadas para pesquisas em turismo de bicicleta
}

\author{
Methodologies used for research in bicycle tourism
}

\section{Camila de Almeida Teixeira}

Mestre em Turismo pela Faculdade de Turismo e Hotelaria da Universidade Federal Fluminense - UFF, Niterói/RJ, Brasil

E-mail: camilateixeira@id.uff.br

\section{Fátima Priscila Morela Edra}

Professora nos curso de graduação e mestrado em Turismo na Faculdade de Turismo e Hotelaria da Universidade Federal Fluminense - UFF, Niterói/RJ, Brasil

E-mail:fedra@id.uff.br 


\section{RESUMO}

Este artigo buscou analisar quais os métodos científicos e os assuntos e temas mais utilizados por pesquisadores em pesquisas sobre o turismo de bicicleta. Para alcançar o objetivo deste trabalho, primeiramente, realizou-se pesquisa em três plataformas de busca, utilizando as palavras-chave cycle tourism, bicycle tourism, bike lane, turismo de bicicleta. Em uma segunda etapa desta seleção foram descartados os artigos que não apresentavam relação com a infraestrutura cicloviária ou relação ao uso da bicicleta como atividade turística. E, no terceiro momento, os artigos foram analisados em relação a sua metodologia. O resultado deste levantamento foi sendo apresentado juntamente com o referencial teórico, seção em que foi explicado de maneira sucinta os tipos de coleta de dados e os tipos de análise de dados utilizados nos artigos selecionados. Posteriormente, pode-se fazer uma reflexão sobre as metodologias encontradas neste lavamento a respeito do turismo de bicicleta e da infraestrutura cicloviária. Esta análise mostrou a existência de diversas metodologias que podem ser utilizadas de diferentes perspectivas sobre um determinado assunto.

Palavras-chave: Análise Metodológica. Infraestrutura Cicloviária. Cicloturismo. Cicloviagens.

\section{ABSTRACT}

This article sought to evaluate the problems and the scientific subjects and the themes most used by researchers in their research. For this work, the first, was done a search in three search platforms, using the keywords cycle tourism, cycling, cycling tourism. In a second stage of this meeting were discarded the articles that were not related to the area of cycling or in relation to the use of the activity as a tourist activity. And, in a third moment, the articles were included in relation to their methodology. The result of this survey was added together with the theoretical reference, the section in which it was explicitly in order to collect the data collection data and the types of analysis data used in the selected data. Subsequently, an analysis can be made on the bases of the evaluation of tourism and cycling road. This analysis has an history in several methods.

Keywords: Methodological analysis. Bicycle infrastructure. Cycle tourism. Cycling tourism. 


\section{INTRODUÇÃO}

O turismo de bicicleta é uma atividade em crescimento e "um modo cada vez mais importante de férias ou férias transporte" (Ritchie, 1998, p. 567). Um dos fatores importantes para o desenvolvimento dessa atividade está relacionado a infraestrutura cicloviária. Ritchie (1998) afirma que em diversas partes do mundo está ocorrendo o desenvolvimento de rotas de bicicleta em estações e linhas férreas desativadas, sendo elas convertidas não somente para ciclistas como também para pedestres.

Além da utilização de linhas férreas desativadas para a utilização de pedestres e ciclistas, há na Europa projetos que estão sendo planejados e implementados como a EuroVelo (Ritchie, 1998), uma rede de ciclovias que passa por 42 países, possui 15 rotas e, quando finalizada, terá mais de 70 mil quilômetros de ciclovias cortando toda a Europa (EuroVelo, 2019). Cidades como Copenhague, onde existe investimento do governo em infraestrutura cicloviária e incentivos para o uso da bicicleta como meio de transporte, apresentam aumento nos usuários de bicicleta e diminuição no uso dos veículos individuais motorizados (Figura 1).

Figura 1 - Tráfego no centro de Copenhague: carro versus bicicleta

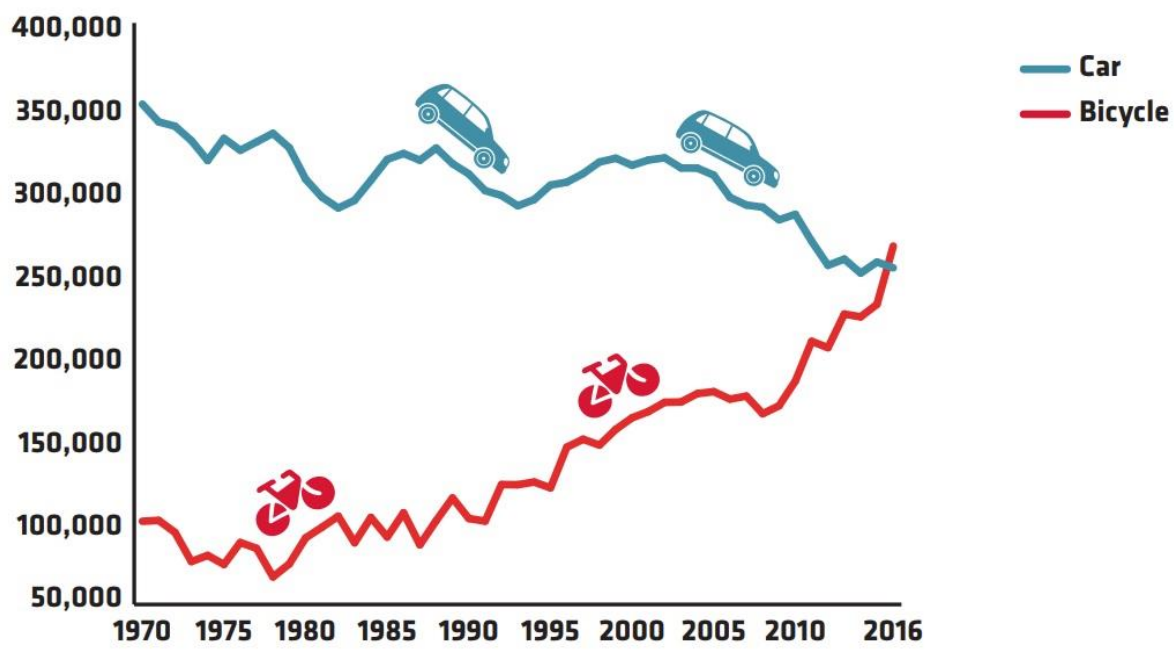

Fonte: City of Copenhagen, 2017.

A figura 1 apresenta a quantidade de veículos individuais motorizados em relação a quantidade de usuários de bicicleta de 1970 a 2010, e projeção de que o número de ciclistas ultrapasse os motoristas até 2016, porém, novo levantamento para verificar se o objetivo foi alcançado ainda não foi divulgado.

A infraestrutura desenvolvida para uso dos residentes, tais como o transporte público, pode ser utilizada por turistas. No caso do cicloturismo urbano, quanto melhor e mais segura a 
infraestrutura, mais os turistas se sentem motivados a utilizar a bicicleta. Já no caso das cicloviagens, estas parecem ter uma configuração diferente na maneira como se dá o consumo do espaço, pois a infraestrutura não impede, necessariamente, na escolha de se fazer uma viagem de bicicleta ou não.

Ritchie (1998) observa que para se ter uma oferta turística sustentável, para satisfazer o turista, se faz necessário a realização de mais pesquisa sobre a demanda do turismo de bicicleta. Talvez a necessidade de mais pesquisas, como citou o autor, possa estar atrelada ao fato de ser uma atividade recente e em crescimento.

Quase duas décadas depois, Saldanha (2017, p. 30) descreve sobre a "escassez de produção acadêmica e institucional para fundamentar novos estudos e aplicações na prática" sobre a interface da bicicleta e turismo. Para o autor, foi a partir de 2015 que houve aumento significativo das publicações.

Portanto, verificar o que está sendo publicado, quais os assuntos e quais as vertentes estão sendo pesquisadas em relação não somente ao turismo de bicicleta, mas também sobre infraestrutura cicloviária, pode contribuir para pesquisas futuras.

Assim, o objetivo desta pesquisa foi analisar os métodos científicos e os assuntos e temas mais utilizados por pesquisadores em pesquisas sobre o turismo de bicicleta.

\section{METODOLOGIA}

A pesquisa bibliográfica utiliza materiais como livros e artigos científicos, alguns estudos exploratórios "podem ser definidos como pesquisas bibliográficas, assim como certo número de pesquisas desenvolvidas a partir da técnica de análise de conteúdo" (Gil, 2008, p. $50)$.

Segundo Lima e Mioto (2007), a pesquisa bibliográfica, permite a utilização de dados dispersos de diferentes tipos de publicações como periódicos e livros. Gil (2008, p. 50) afirma que uma das vantagens deste tipo de pesquisa está no fato de "permitir ao investigador a cobertura de uma gama de fenômenos muito mais ampla do que aquela que poderia pesquisar diretamente". Para Veal (2011, p. 91), a pesquisa bibliográfica "se refere ao processo de identificar e estudar pesquisas anteriormente publicadas".

Assim, esta pesquisa usou a bibliografia para investigar as metodologias utilizadas em publicações de turismo de bicicleta e infraestrutura cicloviária. E, para verificar os principais métodos utilizados, optou-se, primeiramente, por efetuar corte temporal considerando somente as publicações nos últimos cinco anos, decisão que teve por base dois aspectos: (i) padrão temporal adotado por uma convenção da academia de modo geral; (ii) tratar-se do mesmo ano 
(a partir de 2015) em que Saldanha (2017) afirma ter ocorrido aumento significativo das publicações relacionando bicicleta e turismo.

Ademais, limitou-se às bases de dados nos sites Web of Science, Elsevier e publicações em turismo com a busca por palavras chaves que alcançassem uma diversidade de assuntos sobre o tema: bicycicle tourism, cycle tourism, bike lane, bicycle lane, cicloturismo, turismo de bicicleta e ciclovia.

A pesquisa nas bases internacionais utilizando as referidas palavras-chave resultou em 126 artigos e, nas bases nacionais, 21. A fim de refinar o resultado gerado a partir das palavraschave, optou-se, a partir do resumo dos artigos, descartar os artigos que não possuíssem relação com a infraestrutura cicloviária e/ou turismo de bicicleta.

Após a leitura dos resumos dos artigos internacionais, dos 126 artigos encontrados anteriormente, apenas 20 atendiam ao critério definido. E, no caso dos artigos encontrados na base Publicações em Turismo, dos 21, ficaram apenas três. Entretanto, durante a revisão, quatro dos artigos selecionados na base nacional não estava mais disponível sendo excluídos. Assim, para esta pesquisa, foram utilizados 19 artigos científicos.

\section{REFERENCIAL TEÓRICO}

Esta seção tratará das metodologias encontradas nos artigos selecionados. Visando melhor clareza nos resultados trabalhados, eles serão apresentados concomitantemente aos conceitos sobre tipos de pesquisa, estratégias de pesquisa, técnicas/instrumentos de coleta de dados e recursos tecnológicos divididos em subseções.

Antes de iniciar a discussão sobre metodologias, cabe verificar qual a origem das pesquisas, que países são representados pelos pesquisadores? Na Figura 2, onde se mostra a relação de autores por países com base na localização da universidade de origem e o ano de publicação, pode-se perceber uma maior concentração das pesquisas na Europa.

Figura 2 - Quantidade de autores por país e ano

\begin{tabular}{|clc|}
\hline \multirow{3}{*}{ Ano } & País de origem & Quantidade \\
\hline & Dinamarca & 4 \\
& Brasil & 3 \\
& Itália & 2 \\
& Portugal & 1 \\
& França & 1 \\
& Irlanda & 1 \\
& Tailândia & 4 \\
\hline
\end{tabular}




\begin{tabular}{|cll|}
\hline 2016 & Dinamarca & 2 \\
& Suécia & 2 \\
& Itália & 7 \\
& Estados Unidos & 3 \\
& Canadá & 2 \\
& Coréia do Sul & 2 \\
& China & 1 \\
2018 & Estados Unidos & 7 \\
& Dinamarca & 4 \\
& Alemanha & 5 \\
& Brasil & 4 \\
\hline
\end{tabular}

Fonte: Elaborado pelos autores, 2020.

Numa outra perspectiva, ao se ignorar o ano de publicação e se concentrar no quantitativo, verifica-se que apesar dos Estados Unidos estarem no continente americano, apresenta a mesma quantidade de pesquisas que a Dinamarca, dez cada um (Figura 3). Ademais, que o Brasil não se mostra tão distante desse resultado, visto que possui apenas três publicações a menos totalizando sete e apresentando apenas a Itália a sua frente com nove publicações.

Figura 3 - Quantidade de pesquisas por país

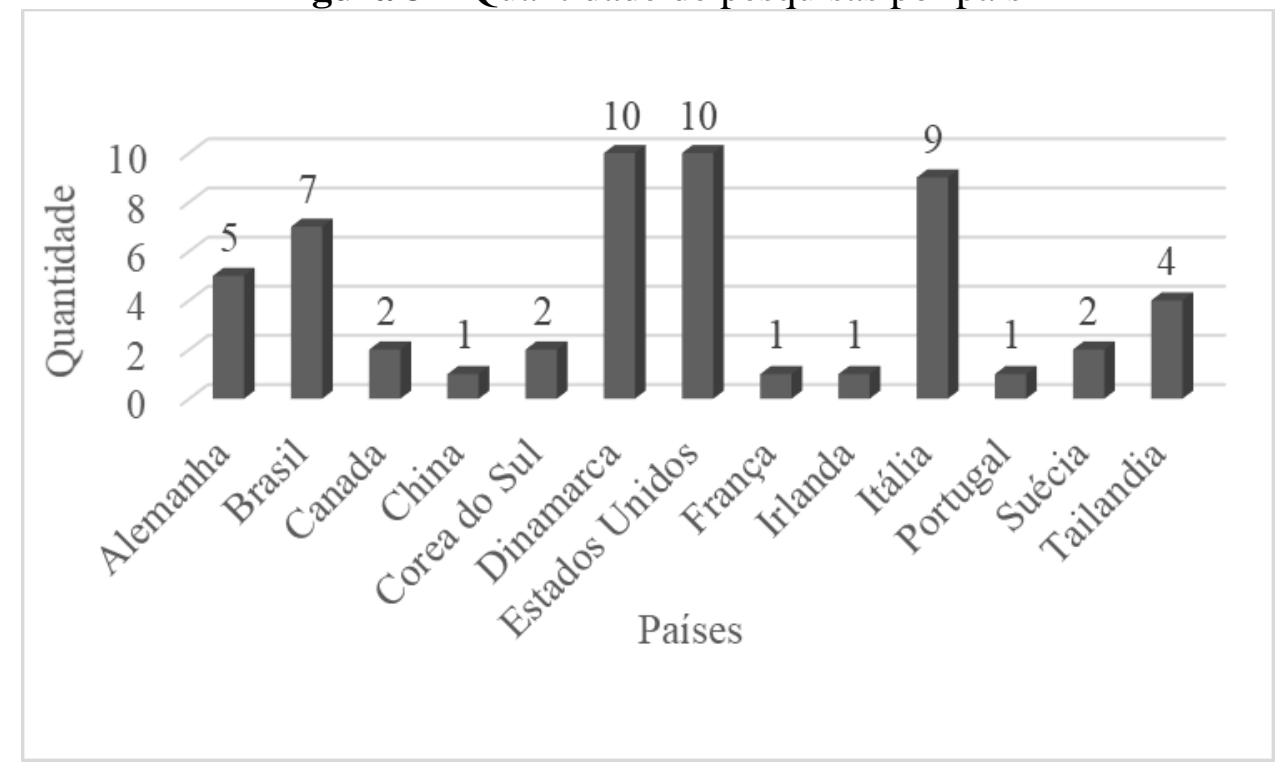

Fonte: Elaborado pelos autores, 2020.

As subseções seguintes trazem conceitos dos tipos de pesquisa, estratégias de pesquisas, técnicas/instrumentos de coletas de dados e recursos tecnológicos para análises estatísticas, léxicas e de conteúdo encontrados nos artigos selecionados, com respectivos artigos que fizeram uso desses. 


\subsection{Tipos de pesquisa}

A classificação dos artigos em relação ao tipo de pesquisa se baseou em Veal (2011), autor que possui estudo focado no lazer e turismo, e em Gil (2008), cientista social com publicações referentes a metodologias de pesquisa na área da ciência social. Para eles, os tipos de pesquisas podem ser:

(i) Descritivas:

(a) visam estudar as características de uma população, levantamento de opinião (Gil, 2008);

(b) pelo fato de o campo de estudos de turismo ser recente, existe "a necessidade de mapear o território". Este tipo de pesquisa pode ser "considerado exploratória: procura descobrir, descrever ou mapear padrões de comportamento em áreas ou atividades que não foram previamente estudadas". Veal (2011) resume este tipo de pesquisa com a função de “investigar, descrever o que é” (2011, p. 29).

(ii) Explicativas:

(a) "Têm como preocupação central identificar os fatores que determinam ou que contribuem para a ocorrência dos fenômenos". A pesquisa explicativa, explica o porquê e a razão das coisas (Gil, 2008, p. 28);

(b) para a pesquisa explicativa, Veal (2011, p. 29) afirma que sua função é “explicar como ou por que as coisas são como são (e usar essa informação para previsões)". Este tipo de pesquisa não possui apenas como objetivo descrever e tentar esclarecer, mas também compreender as causas e, assim, podendo ser utilizada em previsões.

(iii) Avaliativas:

(a) possui como função "avaliar políticas e programas" (Veal, 2011, p. 29).

(iv) Exploratórias:

(a) "Têm como principal finalidade desenvolver, esclarecer e modificar conceitos e ideias" (Gil, 2008, p. 27). A pesquisa exploratória, normalmente, é escolhida quando o campo de estudo é pouco estudado, e envolve 
levantamento bibliográfico e documental, entrevistas não padronizadas ou estudos de caso.

$\mathrm{Na}$ figura 4 se apresentam os tipos de pesquisas verificadas nos artigos selecionados considerando as definições de Gil (2008) e Veal (2011). Interessante observar que, embora Ritchie (1998) indique a necessidade de mais pesquisas sobre o turismo de bicicleta e mesmo tendo se passado duas décadas ainda não se observe o tema com conceitos consolidados, não foi identificada nenhuma pesquisa exploratória. No que se refere aos demais tipos de pesquisa, descritiva, explicativa e avaliativa, as mesmas aparecem de forma equilibrada.

\begin{tabular}{|cc|}
\hline Figura 4 - Tipo de pesquisa \\
\hline Tipo de pesquisa & Quantidade \\
\hline Explicativa & 7 \\
\hline Descritiva & 6 \\
\hline Avaliativa & 6 \\
\hline
\end{tabular}

Fonte: Elaborado pelos autores, 2020.

\subsection{Estratégias de pesquisa}

Verificou-se que os autores utilizaram variadas estratégias em uma mesma pesquisa. Em um panorama geral, os artigos apresentaram estudo de caso e pesquisa documental. São descritos, nas subseções seguintes, o conceito de cada um seguindo a teoria, principalmente, de Veal (2011) e Costa (2005), com posterior apresentação dos artigos que as utilizaram.

\subsubsection{Estudo de caso}

Estudos de caso, normalmente, são utilizados para investigar o porquê ou como determinados eventos acontecem, quando o pesquisador tem pouco controle sobre esses eventos ou "quando o foco se encontra em fenômenos contemporâneos inseridos em algum contexto da vida real" (Yin, 2003, p. 19).

Para Veal (2011), este tipo de pesquisa implica em um estudo de um exemplo, um fenômeno que está sendo pesquisado, tentando compreender este fenômeno como único. $\mathrm{O}$ autor ainda acrescenta que um dos principais aspectos do estudo de caso é a utilização de diferentes tipos de dados e de análises.

Este tipo de método foi identificado em apenas um artigo, o de Giovannini, Malucelli e Nonato (2015) intitulado Designing single origin-destination itineraries for several classes of cycle-tourists. Este estudo de caso se centraliza na cidade de Trebon, na região Boémia do Sul, 
República Tcheca. O trabalho apresenta, como problemática, os recursos limitados e dificuldade das autoridades em administrar estes poucos recursos na implantação de ciclovias para a utilização por cicloturistas e cicloviajantes. Esta pesquisa propôs medidas para solucionar o problema.

\subsubsection{Pesquisa documental}

A pesquisa documental pode ser considerada como dados em "forma de documentos, como livros, jornais, papéis oficiais, registros estatísticos, fotos, discos, filmes e vídeos" (Gil, 2008, p. 147).

Os pesquisadores também têm utilizado recursos audiovisuais como fonte de dados para suas pesquisas. E, por causa das novas tecnologias de registro, como as fotografias, filmes e vídeos, e o aumento nas formas de acesso a esses dados, houve a necessidade de estudos focados nessas fontes, uma vez que ela também é uma linguagem que pode ser interpretada (Costa, 2005).

Alguns pesquisadores utilizaram de recursos documentais para coletar dados e informações para suas pesquisas. No total, foram cinco artigos que utilizaram diferentes fontes documentais (Figura 6). Vale ressaltar, ainda, que nem todos os artigos utilizaram apenas um método para a coleta de dados, alguns utilizaram métodos múltiplos.

Figura 6 - Tipos de fontes documental

\begin{tabular}{|c|c|}
\hline Autores & Tipo de documento \\
\hline Edra, Costa e Fernandes (2015) & Documentos oficiais da prefeitura de Niterói, Rio de Janeiro. \\
\hline Miller e Coutts (2018) & $\begin{array}{l}\text { Plano de mobilidade da cidade, documentos de projeto de bicicleta } \\
\text { (sendo eles relatórios de comitê, memorandos, arquivos de } \\
\text { apresentação pública e documentos de construção), sites da cidade } \\
\text { e artigos de notícias. }\end{array}$ \\
\hline $\begin{array}{l}\text { Griswold, Yu, Filingeri, Grembek e } \\
\text { Walkerd (2018) }\end{array}$ & $\begin{array}{l}\text { Imagens do Google Earth, imagens do Google Maps e gravações } \\
\text { em vídeo. }\end{array}$ \\
\hline $\begin{array}{l}\text { DeCastro, Saldanha, Freitas e } \\
\text { Balassiano (2018) }\end{array}$ & $\begin{array}{l}\text { Documentos que permitissem o levantamento sobre a malha } \\
\text { cicloviária, do Plano Diretor Cicloviário e da existência de } \\
\text { sistemas de bicicleta compartilhada, documentos e dados oficiais } \\
\text { de turismo e outros documentos disponíveis na internet. }\end{array}$ \\
\hline
\end{tabular}

Fonte: Elaborado pelos autores, 2020. 


\subsection{Técnicas/instrumentos de coletas de dados}

Observou-se a utilização, pelos autores, de diferentes técnicas/instrumentos de coleta de dados. De maneira geral, os artigos fizeram uso de questionário, entrevista e observação participante. Em seguida, se apresentam os conceitos de cada um com base nos principais teóricos sobre o tema e os artigos que as empregaram.

\subsubsection{Questionários}

Os questionários são recomendados em pesquisas em que o pesquisador não possui alguns dados ou quando se deseja obter informações "de determinados aspectos da população, opiniões e comportamentos da população" (Costa, 2005, p. 355). As perguntas inseridas no questionário podem ser abertas, quando o respondente pode responder livremente o que quiser sem que haja respostas predefinidas para escolher, ou fechadas, que são perguntas de múltipla escolha (Costa, 2005) em que o respondente deve assinalar uma das alternativas. Há também a possibilidade de combinar as perguntas abertas e fechadas no mesmo questionário (Veal, 2011).

Dos artigos selecionados, seis deles utilizaram questionário, fosse ele aberto, fechado ou misto, são eles:

(i) A new approach to understand modal and pedestrians route in Portugal (Barros, Martínez \& Viegas, 2015);

(ii) Do tourists value different levels of cycling infrastructure (Deenihan \& Caulfield, 2015);

(iii) Intentions to use bike-sharing for holiday cycling: An application of the Theory of Planned Behavior (Kaplan, Manca, Nielsen, \& Prato 2015);

(iv) Measuring the motivation to ride bicycles for tourism through a comparison of tourist attractions (Watthanaklang, Ratanavaraha, Chatpattananan \& Jomnonkwao, 2016)

(v) An empirical analysis of bike sharing usage and rebalancing: Evidence from Barcelona and Seville (Faghih-Imani, Hampshire, Marla \& Elurud, 2017);

(vi) A behavioral modeling approach to bicycle level of service (Griswold et al., 2018)

Nos questionários também podem ser colocadas perguntas com escalas onde o respondente escolhe a resposta de acordo com o tipo de escala utilizada no questionário, um 
exemplo são as pesquisas de satisfação. Veal (2011, p. 354) explica que "por meio dessa técnica, os respondentes são solicitados a indicar se concordam ou não com alguma afirmativa ou a importância que atribuem ao fator, usando um conjunto padrão de respostas". A escala foi utilizada em três artigos:

- Bicycle-friendly infrastructure planning in Beijing and Copenhagen - between adapting design solutions and learning local planning cultures (Zhao, Carstensen, Nielsen \& Olafsson, 2018), utilizou a escala Likert;

- Bike-traveling as a growing phenomenon: Role of attributes, value, satisfaction, desire, and gender in developing loyalty (Han, Meng \& Kim, 2017), utilizou sete tipos de escalas;

- Measuring the motivation to ride bicycles for tourism through a comparison of tourist attractions (Watthanaklang et al., 2016), utilizou escala de avaliação.

\subsubsection{Entrevistas}

Quando a pesquisa bibliográfica e a pesquisa observacional não conseguem oferecer dados suficientes, o pesquisador recorre à entrevista como forma de complementar seus dados (Boni \& Quaresma, 2005). As entrevistas, geralmente gravadas e transcritas para a análise, têm como objetivo obter dados, além de exigir "a formulação prévia das questões" (Costa, 2005, p. 356).

Para este tipo de coleta, o pesquisador escolhe os entrevistados por meio do método de amostragem. Este é considerado por Costa (2005) como um processo de seleção de uma determinada população para uma pesquisa e que pode ser: aleatória, a partir de sorteio, por exemplo; proporcional, quando se conhece o quantitativo populacional e assim se determina a quantidade de entrevistados; ou por conglomerado, definidos e escolhidos por regiões geográficas.

Boni e Quaresma (2005) apresentam seis tipos de entrevista: (i) estruturada; (ii) semiestruturada; (iii) projetiva; (iv) aberta; (v) com grupo focal; e (vi) história de vida. Nos artigos selecionados, foram identificados sete artigos que fizeram uso dos três primeiros tipos de entrevistas. Estas, são explicadas, na figura 5, assim como também são apresentados os artigos selecionados que as utilizaram. 
Figura 5 - Descrição do tipo de entrevista

\begin{tabular}{|c|c|c|}
\hline Projetiva & $\begin{array}{l}\text { Esta entrevista utiliza recursos visuais, } \\
\text { assim é possível aprofundar mais } \\
\text { sobre o assunto pesquisado (Boni \& } \\
\text { Quaresma, 2005). }\end{array}$ & $\begin{array}{l}\text { 1. Do tourists value different levels of cycling } \\
\text { infrastructure? (Deenihan \& Caulfield, 2015); }\end{array}$ \\
\hline Estruturada & $\begin{array}{l}\text { As perguntas para este tipo de } \\
\text { entrevista são previamente formuladas } \\
\text { e dificilmente a entrevista sai do } \\
\text { roteiro das perguntas formuladas } \\
\text { (Boni \& Quaresma, 2005). }\end{array}$ & $\begin{array}{l}\text { 2. A behavioral modeling approach to bicycle } \\
\text { level of service (Griswold et al., 2018); } \\
\text { 3. Bike-traveling as a growing phenomenon: } \\
\text { Role of attributes, value, satisfaction, desire, } \\
\text { and gender in developing loyalty (Han, Meng, } \\
\text { \& Kim, 2017); } \\
\text { 4. Intentions to use bike-sharing for holiday } \\
\text { cycling: An application of the Theory of } \\
\text { Planned Behavior (Kaplan et al., 2015); } \\
\text { 5. Measuring the motivation to ride bicycles for } \\
\text { tourism through a comparison of tourist } \\
\text { attractions (Watthanaklang et al., 2016) }\end{array}$ \\
\hline Semiestruturada & $\begin{array}{l}\text { Este tipo de entrevista mescla } \\
\text { perguntas abertas e fechadas, sendo } \\
\text { possível, também, utilizar recursos } \\
\text { visuais (Boni \& Quaresma, 2005). }\end{array}$ & $\begin{array}{l}\text { 6. Enhancing utilitarian cycling: a case study } \\
\text { (Stromberg \& Karlsson, 2016); } \\
\text { 7. Bicycle-friendly infrastructure planning in } \\
\text { Beijing and Copenhagen - between adapting } \\
\text { design solutions and learning local planning } \\
\text { cultures (Zhao et al., 2018); }\end{array}$ \\
\hline
\end{tabular}

Fonte: Elaborado pelos autores, 2020.

\subsubsection{Observação participante}

A observação pode ser caracterizada como uma forma de coleta de dados não invasiva ao observado. Este tipo de coleta apresenta algumas vantagens quando, por exemplo, se deseja estudar o comportamento, pois o sujeito, ao saber que está sendo observado por um pesquisador, pode alterar seu comportamento (Veal, 2011). "A observação é capaz de apresentar uma perspectiva sobre uma situação que não é óbvia para os indivíduos envolvidos” (Veal, 2011, p. 148).

De modo semelhante, Gil (2008, p. 101) afirma que de maneira geral as pessoas, "ao se sentirem observadas, tendem a ocultar seu comportamento, pois temem ameaças à sua privacidade". A alteração no comportamento influencia no resultado da amostra, pois quando se altera a realidade, deixa de ser uma amostra confiável, pois pode estar ocultando algo importante para a pesquisa. 
Há três tipos de observação destacados por Gil (2008): simples; sistemática e; participante. Dentre estes três tipos de observação apenas a observação participante foi encontrada no levantamento metodológico. O autor explica este tipo de observação como:

[...] participação real do conhecimento na vida da comunidade, do grupo ou de uma situação determinada. Neste caso, o observador assume, pelo menos até certo ponto, o papel de um membro do grupo. Daí por que se pode definir observação participante como a técnica pela qual se chega ao conhecimento da vida de um grupo a partir do interior dele mesmo (Gil, 2008, p. 103).

Veal (2011, p. 239) diz que a observação participante ocorre quando o "pesquisador é participante do ambiente em estudo", "torna-se parte do processo social que está sendo estudado" (Veal, 2011, p. 275). A isso, acrescenta-se que, em alguns casos, o observador passa a adotar "temporariamente um estilo de vida que é próprio do grupo que está sendo estudado" (Costa, 1987 como citado em Boni \& Quaresma, 2005).

A observação participante foi utilizada em apenas um artigo intitulado A multiple case study of local and creative financing of bicycle and pedestrian infrastructure, os autores desta pesquisa também utilizaram outros métodos de coleta de dados como o estudo de caso, entrevista com perguntas semiestruturadas e documentos.

\subsection{Recursos tecnológicos utilizados para análise de dados}

A análise de dados se apresenta como parte importante da pesquisa por ser a etapa em que os dados coletados são processados e interpretados. Existem vários tipos de dados que podem ser analisados, tais como imagens, textos, discursos, comportamentos e diversos outros materiais. Os dados podem ser analisados de diferentes maneiras. Com o avanço da tecnologia existem alguns softwares que facilitam a análise de dados, como o Nvivo e o Nlogit, para análise qualitativa. Há também o uso da estatística para analisar dados quantitativos.

\subsubsection{Software de análise}

Existem vários softwares de análise quantitativa de dados, os computer-aided qualitative data analysis software (CAQDAS), como o Nvivo e Nlogit, ambos utilizados para análise no levantamento metodológico. Este tipo de software oferece "a possibilidade de testar e relacionar hipóteses valendo-se dos recursos e benefícios trazidos pela informática, até então

privilégio da pesquisa quantitativa, utilizando qualquer tipo de técnica qualitativa, com qualquer material que possa ser transformado em texto" (Teixeira \& Becker, 2001, p. 95). 
O software Nvivo, segundo (Veal, 2011, p. 290), ajuda na "formatação e na compreensão dos dados", ainda é possível, com este software, "elaborar e testar pressupostos teóricos sobre os dados".

Hensher, Rose e Greene (2015) afirmam que o Nlogit é utilizado em várias disciplinas como as de transporte, economia, agricultura, saúde, marketing, estatística e todas as da área de ciências sociais.

A utilização desses tipos de software ajuda o pesquisador na tarefa de analisar os dados coletados, principalmente quando se tem um grande quantitativo de dados a serem analisados.

$\mathrm{Na}$ análise, foram encontrados dois artigos que utilizaram softwares diferentes. A pesquisa A behavioral modeling approach to bicycle level of service de Griswold et al. (2018), fez o uso do Nvivo, enquanto Deenihan e Caulfield (2015) utilizaram o software Nlogit para analisarem os dados obtidos para a pesquisa Do tourists value different levels of cycling infrastructure?

\title{
3.4.2. Análise estatística
}

Utilizada em pesquisas quantitativas, a análise estatística possui abordagem e teste estatístico, de acordo com Veal (2011). A abordagem estatística pode ser: estimativa probabilística, distribuição normal, demonstração de resultados, significância, hipótese nula e variáveis dependentes e independentes. Esta última, normalmente, é utilizada para verificar a existência de relação entre a variável dependente e a(s) variável(is) independente(s). Quando se verifica relação entre elas, isso quer dizer que "a variável independente influencia a variável dependente" (Veal, 2011, p. 452).

Oliveira Filho (2002, p. 3) explica de forma clara este tipo de análise:

\begin{abstract}
A análise de regressão tem por objetivo descrever através de um modelo matemático, as relações existentes entre duas ou mais variáveis, a partir de $n$ observações dessas variáveis. As variáveis independentes que são acrescentadas ao modelo têm como finalidade melhorar a capacidade de predição em confronto com a regressão linear simples. (Oliveira Filho, 2002, p. 3).
\end{abstract}

Na pesquisa de Faghih-Imani et al. (2017), An empirical analysis of bike sharing usage and rebalancing: Evidence from Barcelona and Seville, foi utilizada a regressão linear em parte da pesquisa para análise de dados. 


\section{DISCUSSÃO SOBRE AS METODOLOGIAS UTILIZADAS}

Os dados levantados mostraram que pesquisas sobre infraestrutura cicloviária e sobre turismo de bicicleta utilizam diversos métodos, assim necessitando de levantamentos primários.

Na figura 7 se apresentam os assuntos contemplados assim como a quantidade de vezes observadas.

Figura 7 - Assuntos abordados

\begin{tabular}{|lc|}
\hline \multicolumn{1}{|c|}{ Assunto } & Quantidade \\
\hline Planejamento e infraestrutura cicloviária. & 12 \\
\hline Escolha do meio de transporte. & 3 \\
\hline Desenvolvimento do turismo de bicicleta. & 2 \\
\hline Políticas públicas para o desenvolvimento de infraestrutura cicloviária. & 1 \\
Compartilhamento de bicicleta. & 1 \\
\hline
\end{tabular}

Fonte: Elaborado pelos autores, 2020.

$\mathrm{O}$ assunto mais pesquisado trata do planejamento e infraestrutura cicloviária. Porém, antes de tratar sobre o planejamento, devem-se compreender as políticas locais para que possa ser feito o planejamento bem estruturado e, assim, desenvolver a infraestrutura cicloviária de maneira adequada para seus usuários, sejam eles residentes ou turistas.

A fim de ilustrar melhor o exposto na seção anterior, a Figura 8 apresenta, de maneira resumida, as metodologias utilizadas pelos pesquisadores para o desenvolvimento dos artigos.

Figura 8 - Resumo dos tipos de coleta de dados por autor(es)

\section{Autor(es)/ano}

Barros e Martínez e Viegas (2015)

Bernardi e Rupi (2015)

Bogdanović, Basarić, Ruškić e Garunović (2016)

DeCastro et al. (2018)

Deenihan e Caulfield (2015)

Edra, Costa e Fernandes (2015)

Faghih-Imani et al. (2017)

Giovannini, Malucelli e Nonato (2017)

Greibe e Buch (2016)

Griswold et al. (2018)

Han, Meng e Kim (2017)

Kaplan et al. (2015)

Malucelli, Giovannini e Nonato (2015)
Métodos/recursos utilizados

Survey, questionário estruturado.

Pesquisa de campo e análise documental.

Pesquisa documental.

Estudo de caso.

Entrevista projetiva.

Análise documental

Entrevista e pesquisa de campo.

Estudo de caso.

Vídeo.

Questionário semiestruturado, imagem, observação

participante e entrevista.

Questionário estruturado com escala.

Questionário estruturado.

Observação. 


\begin{tabular}{|ll|}
\hline Miller e Coutts (2018) & Estudo de caso. \\
\hline Ruocco, Sicignano, Fiore e D’Andria (2017) & Pesquisa documental. \\
$\begin{array}{l}\text { Schleinitz, Petzoldt, Kröling, Gehlert e Mach } \\
\text { (2019) }\end{array}$ & Imagem. \\
\hline $\begin{array}{l}\text { Strömberg e Karlsson (2016) } \\
\text { Watthanaklang et al. (2016) }\end{array}$ & Estudo de caso, entrevista e questionário semiestruturado. \\
\hline Zhao et al. (2018) & Questionário estruturado. \\
\hline
\end{tabular}

Fonte: Elaborado pelos autores, 2020.

O que se pode perceber neste estudo é a variedade de metodologias para pesquisas sobre bicicleta e turismo. Porém, percebe-se que há poucos estudos relacionando a infraestrutura cicloviária com o turismo, principalmente, no que se refere a viagens de bicicleta. Por ser um assunto pouco trabalhado, existe a carência de informações sobre, forçando pesquisas em que exija, primeiramente, a familiarização com o campo estudado, para que posteriormente possam ser feitas pesquisas mais sólidas.

\section{CONSIDERAÇÕES FINAIS}

O levantamento bibliográfico, verificou quais os tipos de metodologia foram mais utilizados nos últimos cinco anos (2015-2019). Ainda que os assuntos sobre turismo de bicicleta e infraestrutura cicloviária sejam limitados, as metodologias são diversas.

Como esta pesquisa mostrou que existe maior quantitativo de estudos relacionados ao planejamento e infraestrutura cicloviária, nota-se uma carência em outros assuntos que também são relevantes e que precisam de mais estudos como as políticas públicas em paralelo ao planejamento para o desenvolvimento do turismo de bicicleta. E no que se refere à metodologia, esta deve ser utilizada de acordo com o tipo de abordagem sobre o assunto.

Sugerem-se pesquisas que identifiquem o perfil dos cicloturistas urbanos e dos cicloviajantes, pesquisas questionando sobre semelhanças e diferenças entre cicloturistas e cicloviajantes, tais como respectivas maneiras de viajar.

Em relação à metodologia, também se sugere a utilização de conteúdos disponíveis pelos próprios cicloviajantes, por exemplo. Visto que o conteúdo disponibilizado por este tipo de turista, geralmente, é em forma de vídeos por meio do Youtube, fotografias por meio do Instagram ou por meio de relatos em formatos de livros e blogs. Para a análise deste tipo de conteúdo podem ser utilizados softwares como os apresentados neste trabalho para facilitar o processamento de dados. 


\section{AGRADECIMENTO}

Ao Conselho Nacional de Desenvolvimento Científico e Tecnológico - CNPq pela concessão de bolsa de pesquisa.

\section{REFERÊNCIAS}

Barros, A. P., Martínez, L. M., \& Viegas, J. M. (2015). A new approach to understand modal and pedestrians route in Portugal. Transportation Research Procedia, Delft, 10(1), 860-869.

Bernardi, S., \& Rupi, F. (2015). An analysis of bicycle travel speed and disturbances on offstreet and on-street facilities. Transportation Research Procedia, 5(1), 82-94.

Bogdanović, V., Basarić, V., Ruškić, N., \& Garunović, N. (2016). Study of the establishment of the regional cycling route Srem. Transportation Research Procedia, 14, 2334-2343.

Boni, V., \& Quaresma, S. J. (2005). Aprendendo a entrevistar: como fazer entrevistas em Ciências Sociais. Revista Eletrônica dos Pós-graduandos em Sociologia Política da UFSC, Santa Catarina, 2(13), 68-80. Recuperado de https://periodicos.ufsc.br/index.php/emtese/article/view/18027/16976.

City of Copenhagen. (2017). Copenhagen: City of cyclists. Recuperado de http://www.cycling-embassy.dk/wp-content/uploads/2017/07/Velo-city_handout.pdf.

Costa, C. (2005). Sociologia: Introdução à ciência da sociedade. São Paulo: Moderna.

DeCastro, J., Saldanha, L., Freitas, L., \& Balassiano, R. (2018). Políticas cicloinclusivas no planejamento do turismo no Brasil: um estudo sobre as cidades-sede da Copa do Mundo FIFA 2014. Revista Acadêmica: Observatório de inovação do turismo, 12(1), 66-85.

Deenihan, G., \& Caulfield, B. (2015). Do tourists value different levels of cycling infrastructure? Tourism Management, 46, 92-101.

Edra, F. P. M., Costa, M. L., \& Fernandes, T. T. (2015). Cicloturismo em Niterói: potencialidade a partir do Rio de Janeiro. Caderno Virtual do Turismo, 15(3), 331-345.

EuroVelo. (2019). Discover Europe by bike! Recuperado de http://www.eurovelo.com/en.

Faghih-Imani, A., Hampshire, R., Marla, L., \& Elurud, N. (2017). An empirical analysis of bike sharing usage and rebalancing: Evidence from Barcelona and Seville. Transportation Research Part A: Policy and Practice, 97(1), 177-191.

Giovannini, A., Malucelli, F., \& Nonato, M. (2017). Cycle-tourist network design. Transportation Research Procedia, 22(1), 154-163.

Gil, A. C. (2008). Métodos e Técnicas de pesquisa social. São Paulo: Editora Atlas.

Greibe, P., \& Buch, T. S. (2016). Capacity and Behaviour on One-way Cycle Tracks of Different Widths. Transportation Research Procedia, 15, 122-136. 
Griswold, J. B., Yu, M., Filingeri, V., Grembek, O., \& Walkerd, J. L. (2018). A behavioral modeling approach to bicycle level of service. Transportation Research Part A: Policy and Practice, 116, 166-177.

Han, H., Meng, B., \& Kim, W. (2017). Bike-traveling as a growing phenomenon: Role of attributes, value, satisfaction, desire, and gender in developing loyalty. Tourism Management, $59,91-103$.

Hensher, D. A., Rose, J. M., \& Greene, W. H. (2015). Nlogit for applied choice analysis. In: Hensher, D. A., Rose, J. M., \& Greene, W. H. Applied Choice Analysis. Cambridge: Cambrigde University Press.

Kaplan, S., Manca, F., Nielsen, T. A. S., \& Prato, C. G. (2015). Intentions to use bike-sharing for holiday cycling: An application of the Theory of Planned Behavior. Tourism Management, 47, 34-46.

Lima, T. C. S., \& Mioto, R. C. T. (2007). Procedimentos metodológicos do conhecimento científico: a pesquisa bibliográfica. Katálysis, 10, 37-45. Recuperado de http://www.scielo.br/scielo.php?script=sci_arttext\&pid=S1414-49802007000300004

Malucelli, F., Giovannini, A., \& Nonato, M. (2015). Designing single origin-destination itineraries for several classes of cycle-tourists. Transportation Research Procedia, 10, 413 422.

Miller, S., \& Coutts, C. (2018). A multiple case study of local \& creative financing of bicycle and pedestrian infrastructure. Case Studies On Transport Policy, 6(2), 257-264.

Oliveira Filho, M. L. (2002). A utilização da regressão linear como ferramenta estratégica para a projeção dos custos produção. Anais do IX Congresso Brasileiro de Custos, São Paulo, SP, Brasil. Recuperado de https://anaiscbc.emnuvens.com.br/anais/article/viewFile/2762/2762.

Ritchie, B. W. (1998). Bicycle tourism in the South Island of New Zealand: planning and management issues. Tourism Management, 19(6), 567-582.

Ruocco, G., Sicignano, E., Fiore, P., \& D’Andria, E. (2017). Sustainable reuse of disused railway. Procedia Engineering, 180, 1643-1652.

Saldanha, L. (2017). Impactos da amostra acadêmica do I Encontro para desenvolvimento do cicloturismo no estado da arte nacional: panorama da literatura nacional. In: Edra, F. P. M., Castro, J., \& Saldanha, L. (Org.). Cicloturismo urbano em foco, Niterói, 30-32. Recuperado de https://pedalufftur.blogspot.com/p/blog-page_9.html.

Schleinitz, K., Petzoldt, T., Kröling, S., Gehlert, T., \& Mach, S. (2019). (E-)Cyclists running the red light: The influence of bicycle type and infrastructure characteristics on red light violations. Accident Analysis And Prevention, 122, 99-107.

Strömberg, H., \& Karlsson, I. M. (2016). Enhancing utilitarian cycling: a case study. Transportation Research Procedia, 14(1), 2352-2361.

Teixeira, A. N., \& Becker, F. (2001). Novas possibilidades da pesquisa qualitativa via sistemas CAQDAS. Sociologias, 5(3), 94-113. Recuperado de http://www.scielo.br/pdf/soc/n5/n5a06.pdf. 
Veal, A. J. (2011). Metodologia de Pesquisa em Lazer e Turismo. São Paulo: Aleph.

Watthanaklang, D., Ratanavaraha, V., Chatpattananan, V., \& Jomnonkwao, S. (2016). Measuring the motivation to ride bicycles for tourism through a comparison of tourist attractions. Transport Policy, 52, 153-163.

Yin, R. K. (2003). Estudo de caso: planejamento e métodos. Porto Alegre: Sage,

Zhao, C., Carstensen. T. A., Nielsen, T. A., \& Olafsson, A. S. (2018). Bicycle-friendly infrastructure planning in Beijing and Copenhagen - between adapting design solutions and learning local planning cultures. Journal of Transport Geography, 68, 149-159.

FORMATO PARA CITAÇÃO DESTE ARTIGO

TEIXEIRA, C. A., \& EDRA, F. P. M. (2021). Metodologias utilizadas para pesquisas em turismo de bicicleta. Revista de Turismo Contemporâneo, 9(2), 306-324.

https://doi.org/10.21680/2357-8211.2021v9n2ID22142 